\title{
Erratum to: Neuropsychiatric Symptoms in Patients with Dementias Associated with Cortical Lewy Bodies: Pathophysiology, Clinical Features, and Pharmacological Management
}

\author{
Clive Ballard · Dag Aarsland · Paul Francis • \\ Anne Corbett
}

Published online: 1 July 2014

(C) Springer International Publishing Switzerland 2014

Erratum to: Drugs Aging (2013) 30:603-611

DOI 10.1007/s40266-013-0092-x

The Acknowledgments section should have read as follows:

$\mathrm{CB}$ and $\mathrm{AC}$ would like to acknowledge the support of the National Institute for Health Research (NIHR) Mental Health Biomedical Research Centre and Dementia Unit at South London and Maudsley NHS Foundation Trust and (Institute of Psychiatry) King's College London. This article presents independent research partially-supported by the National Institute for Health Research (NIHR). The views expressed are those of the author(s) and not necessarily those of the NHS, the NIHR or the Department of Health.

The online version of the original article can be found under doi:10.1007/s40266-013-0092-x.

C. Ballard $(\square)$. P. Francis · A. Corbett

Wolfson Centre for Age-Related Diseases, King's College

London, Wolfson Building, Guy's Campus,

London SE1 1UL, UK

e-mail: clive.ballard@kcl.ac.uk

D. Aarsland

Karolinska Institutet Alzheimer's Disease Research Centre,

Novum, Karolinska Institutet, Stockholm 141 86, Sweden 